\title{
Community-based medication disposal pilot initiative in southwest tribal communities
}

\author{
Isaac Ampadu ${ }^{1 *}$, Robert Morones ${ }^{2}$, Andrea Tsatoke $^{3}$, Lacie Ampadu ${ }^{4}$, Martin Stephens ${ }^{5}$, William C. Crump ${ }^{6}$ and \\ David Bales ${ }^{7}$
}

From National Conference on American Indian and Alaska Native Injury and Violence Prevention: Bridging Science, Practice, and Culture Denver, Colorado. 23-25 July 2019

\begin{abstract}
Background: Misuse and abuse of prescription drugs including opioids has been a driving force behind the drug overdose epidemic plaguing communities across the USA for more than two decades. Medication accumulation in the home environment can contribute to this issue. However, research on proper disposal in rural communities is limited. For this project, an applied public health approach was used to raise awareness and improve prescription drug disposal practices by pilot testing prescription drug disposal systems in participating communities.
\end{abstract}

Methods: A community-based disposal project was facilitated with assistance from community partners. The project centered on distribution of drug deactivation bags in homes and medication drop boxes at multiple healthcare facilities.

Results: The team distributed 215 drug deactivation bags to 162 community households resulting in destruction of 8011 pills, 8 medicated dermal patches and $777 \mathrm{~mL}$ of liquid medication. A total of 4684 pounds of medication were collected and disposed of through healthcare facility drop boxes.

Conclusion: The strategies identified are scalable and easy to replicate to meet any community's needs in reducing potential challenges of medication diversion.

Keywords: Behavior change, Interventions, Public health, Poisoning, Medication disposal, Community, Opioids, Tribal

\section{Background}

Misuse and abuse of prescription drugs, including opioids, has been a driving force behind the overdose epidemic plaguing communities across the nation for more than two decades. As the leading cause of injury-related death, drug overdose claimed the lives of more than 70,000 individuals in 2019 (National Institutes of Health 2020). The impact of prescription drug abuse/misuse

\footnotetext{
*Correspondence: Isaac.ampadu@ihs.gov

1 IHS - Western Arizona District Office, 1553 West Todd Drive, Suite 104, Tempe, AZ 85283, USA
}

Full list of author information is available at the end of the article has been felt across all racial and ethnic groups; however, American Indian/Alaskan Natives (AI/AN) have felt the magnitude of the crisis. In 2018, AI/AN population had the second-highest drug overdose death rate (14.2 deaths/100,000 people) in the USA (Wilson et al. 2020).

Published literature suggests medication left unsecured in the home contributes to an increased risk of intentional medication abuse, theft, diversion by individuals and unintentional poisoning due to ingestion by small children or pets (McCance-Katz 2019).

The US Food and Drug Administration (FDA) provides guidance for proper medication disposal, which includes disposal at drug take-back sites (e.g., retail pharmacies, original author(s) and the source, provide a link to the Creative Commons licence, and indicate if changes were made. The images or other third party material in this article are included in the article's Creative Commons licence, unless indicated otherwise in a credit line to the material. If material is not included in the article's Creative Commons licence and your intended use is not permitted by statutory regulation or exceeds the permitted use, you will need to obtain permission directly from the copyright holder. To view a copy of this licence, visit http://creativecommons.org/licenses/by/4.0/. The Creative Commons Public Domain Dedication waiver (http://creativeco mmons.org/publicdomain/zero/1.0/) applies to the data made available in this article, unless otherwise stated in a credit line to the data. 
police departments), flushing in a domestic sewer system if listed on the FDA approved "Flush List," which includes some medications sought after for their misuse and/or abuse potential, or other types of medications that can be discarded in the domestic trash (Center for Drug Evaluation and Research n.d.).

Due to the remote setting of many tribal communities, literature is limited on barriers and access to proper medication disposal. This often results in stockpiling medications in the home environment or improperly discarding in the trash or toilet. From a previous project survey conducted by the Indian Health Service (IHS) Injury Prevention Program (IPP), only one out of every five individuals who had been prescribed medications disposed of them properly. To address this issue, IHS introduced strategies to improve disposal.

IHS is the principal healthcare provider and health advocate for AI/AN communities, which includes preventative services offered by IPP. IHS consists of 12 geographical areas across the USA; each area supports a unique group of tribes (Service, I. H. 2020). Within IHS Division of Environmental Health Service, IPP is designed to assist tribal communities to reduce injuryrelated risk factors. As of 2018, injuries were the leading cause of AI/AN death among those ages 1-54 (National Center for Injury Prevention and Control, C. n.d.). Through collaborative efforts, IPP utilized an applied public health approach to raise awareness, provide education and improve prescription drug disposal practices by pilot testing two drug disposal systems.

\section{Methods}

The project's goal was to determine if the distribution of drug deactivation bags and medication drop boxes would serve as acceptable options for medication disposal in tribal communities. Both methods met US Drug Enforcement Administration (DEA) requirements. Tribal leadership in participating communities and the appropriate IHS Institutional Review Board formally approved the project.

\section{Baseline assessment}

During routine home safety assessments, medications were frequently observed stockpiled and unsecured throughout the residence. Anecdotal comments were received from tribal elders and other community members expressing the need for education and access to proper medication disposal options. In addition, telephone interviews were conducted by IPP staff to document IHS and tribally operated healthcare facility pharmacy department perspectives to better understand community disposal options.

During interviews, the following were asked:
1. Is education provided to the community regarding proper disposal of expired/unused medications?

2. Is there a location within the healthcare facility for community members to dispose of medications?

3. If there is no system in place, would the facility participate in a project focused on safe medication disposal?

Responses indicated that none of the healthcare facilities had an openly accessible system for collecting and disposing of unused or expired medications. Baseline information suggested accessible options for medication disposal would reduce unused/excess prescription medications in the home environment.

\section{Drug deactivation bags}

Drug deactivation bags were provided to IPP at no additional cost as part of a statewide response to the opioid epidemic; however, there is an average cost of $\$ 3.90$ per bag. Over 1700 bags were received for distribution among tribal communities across Arizona. This initiative was piloted within six communities over a three-month period.

\section{Product description}

Drug deactivation bags selected for this project contained carbon-activated powder that neutralizes medication. Medium-size bags were distributed, which allow for destruction of 45 pills, six fluid ounces of liquid medication, and six dermal patches. Deactivation bags work by depositing medications, adding warm water, sealing, and shaking the bag to mix contents. After $30 \mathrm{~s}$, contents are deactivated and safe for deposit in the domestic trash.

\section{Tools development}

An educational flyer was created to promote participation and provide step-by-step instructions of deactivation bags.

A community partner distributed the flyer and bags along with an in-person overview. A data collection form was developed for use during distribution. The forms gathered the following information: patient's age and quantity of unused/expired medication (whether pills, patches, liquid or other); quantity and medication type being disposed; reason for disposal (i.e., discontinued, expired, unused, or other); and whether medication was prescribed for pain relief. Discontinued medications were classified as: medical provider, original prescriber, instructed individuals to discontinue use; expired medications were those beyond the use-by or expiration date; unused medications were defined as medications individuals elected to stop taking. An individual completing the form could select multiple options as reasons for 
medication disposal, such as expired and discontinued, or unused and expired. Space was provided for both community partners and community members to initial and verify the destruction of medications. A master data collection spreadsheet was created to track usage for all pilot sites.

\section{Distribution}

Pharmacists and community partners distributed drug deactivation bags using multiple methods: (1) during community events, (2) during home or provider office visits, and (3) through door-to-door campaigns. Community partners included Community Health Representatives (CHR) Program and Public Health Nurses (PHN) that provide home health services to community members. The CHR/PHN program is a primary healthcare program that provides services geared toward health promotion, disease prevention, and reducing health risks at the community level for elderly or disabled patients. As part of their role, they advocate for public health at the local level and assist community members with medication distribution.

Prior to implementation, a brief kick-off meeting was held with the community partners to discuss the project goals and deliverables. This meeting also served as a training session to review the project materials, to view a 2-min "how-to" video by the manufacturer, and as an opportunity for hands-on training.

\section{Medication drop boxes}

Baseline assessments revealed IHS and tribally operated healthcare facilities did not have drop boxes for community medication disposal. Although they offered periodic take-back events, they were interested in obtaining a box to expand disposal options for their patients. Twenty-six (26) of the 29 participating sites were in rural setting. This was defined using Rural-Urban Communing Area (RUCA) code methodology (Defining rural population 2021). This portion of the project included healthcare facilities in Arizona, Minnesota, Nevada and Oklahoma.

\section{Product description}

Stainless-steel collection drop boxes with the capacity to hold 18 to 36 gallons were selected for the project. The box secures to the floor or wall and is equipped with two locks on the main door and a one-way medicine drop. Each box included a removable, prepaid, ship-back liner. When full, liners must be sealed and returned to the vendor via common carrier to undergo proper destruction. Boxes for this project were purchased through approved government vendors and cost between $\$ 1300$ and $\$ 1450$ per unit. Annual maintenance, which included liner and common carrier fee, ranged from $\$ 465$ to $\$ 675$. Drop box site installations were in accordance with Title 21 Code of Federal Regulation Part 1317 Subpart B enforced by DEA. Requirements included: (1) a DEA license, (2) 24-h monitoring and (3) bolted/secured to floor or wall.

\section{Tools development}

A flyer was developed and provided to points of contact (POC) highlighting the importance of the medication drop box, benefits to the community and the facility, and DEA requirements for collection sites. A centralized tracking spreadsheet was developed to analyze disposal data made available by the manufacturer through an online portal.

\section{Distribution}

Funding for drop boxes was site-specific. Chief Pharmacists were the facility POCs and were responsible for obtaining funding for the drop box and its maintenance.

\section{Results}

Key findings from this pilot project included the widespread distribution of medication deactivation bags in conjunction with increased public knowledge and awareness of medication drop box locations.

\section{Drug deactivation bags}

In May 2019, the IPP collaborated with five Arizona tribal communities and distributed 215 drug deactivation bags to 162 community households. These bags were used to destroy 8011 pills, $777 \mathrm{~mL}(\mathrm{~mL})$ of liquid, and eight medicated dermal patches (Table 1).

Unused medications were the leading reason for disposal, followed by discontinued medications. Of the 8011 pills destroyed, 1054 were unused; 862 were discontinued; 221 were disposed due to being expired; 359 were a combination of being discontinued, expired, or unused; and, 246 were due to "other" reasons not listed. Of the $777 \mathrm{~mL}$ liquids, $713 \mathrm{~mL}$ were neutralized due to discontinued use, and $64 \mathrm{~mL}$ were disposed of for varied reasons (e.g., discontinued and expired). All eight patches were destroyed due to discontinued use (Fig. 1).

\begin{tabular}{ll}
$\begin{array}{l}\text { Table } 1 \text { Forms of medications } \\
\text { deactivation bags }\end{array}$ & disposed using the \\
\hline Types of medications disposed & $\begin{array}{l}\text { Number of } \\
\text { medications } \\
\text { disposed }\end{array}$ \\
\hline Pills & 8011 \\
Patches & 8 \\
Liquid & $777 \mathrm{~mL}$ \\
\hline
\end{tabular}




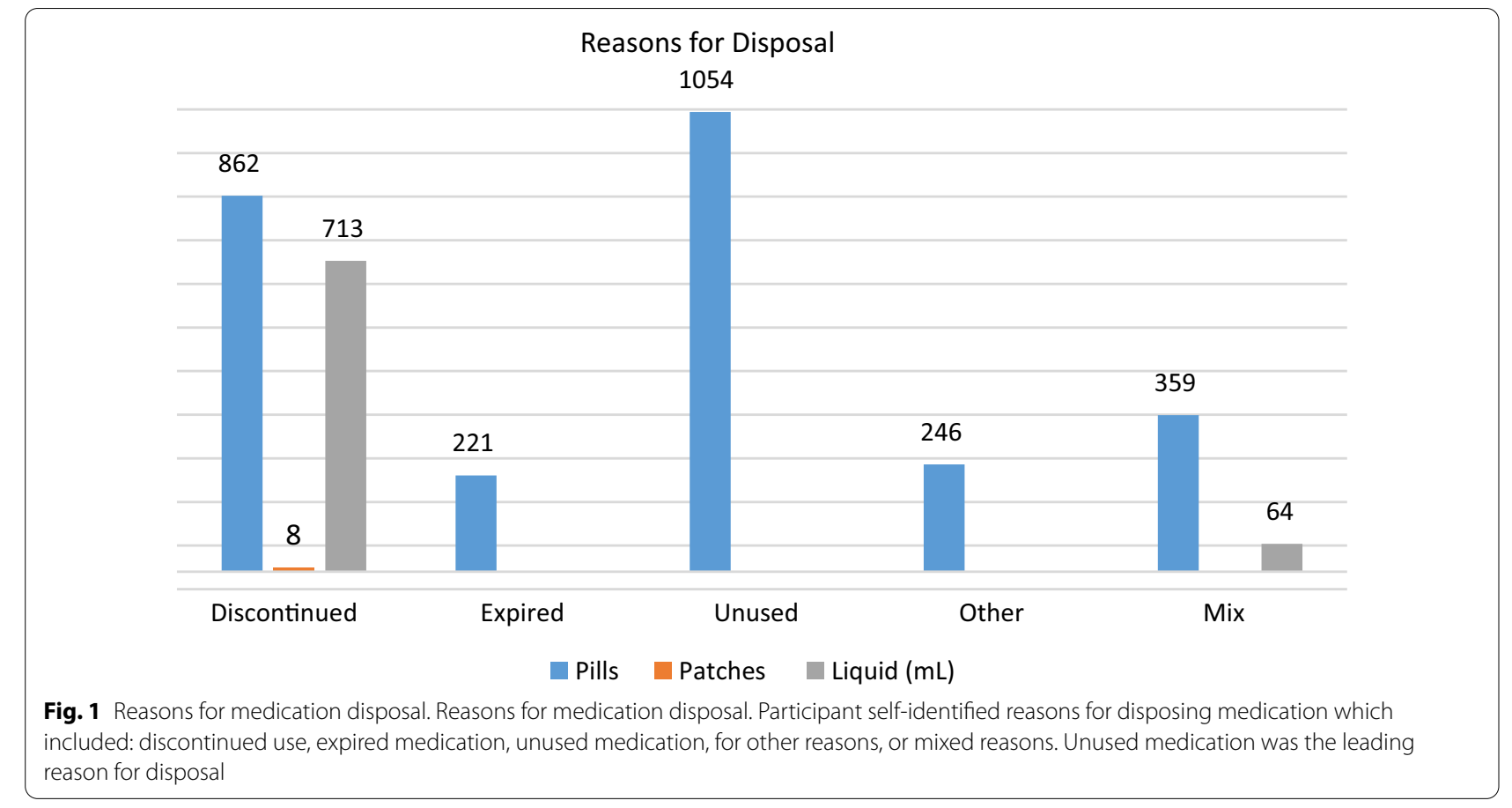

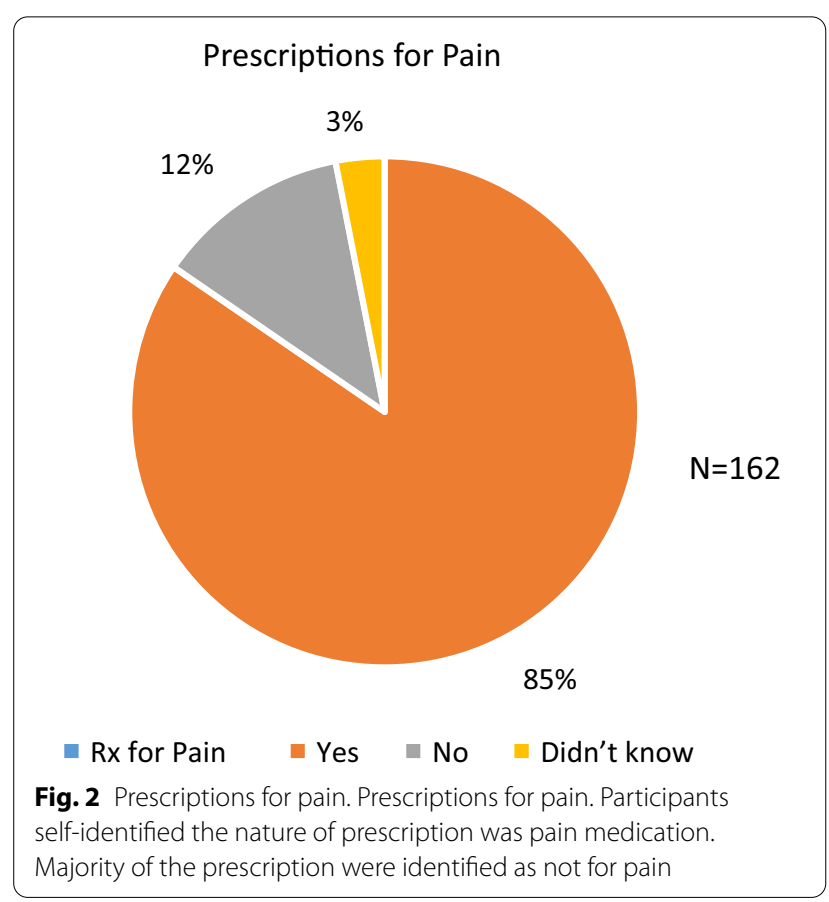

Eighty-five percent $(n=137)$ of households reported "Yes" that they were disposing of prescription pain medication, while $12 \%(\mathrm{n}=20)$ reported "No" to disposing of pain medication; and $3 \%(n=5)$ reported, "Didn't Know" if they were disposing of pain medication (Fig. 2).

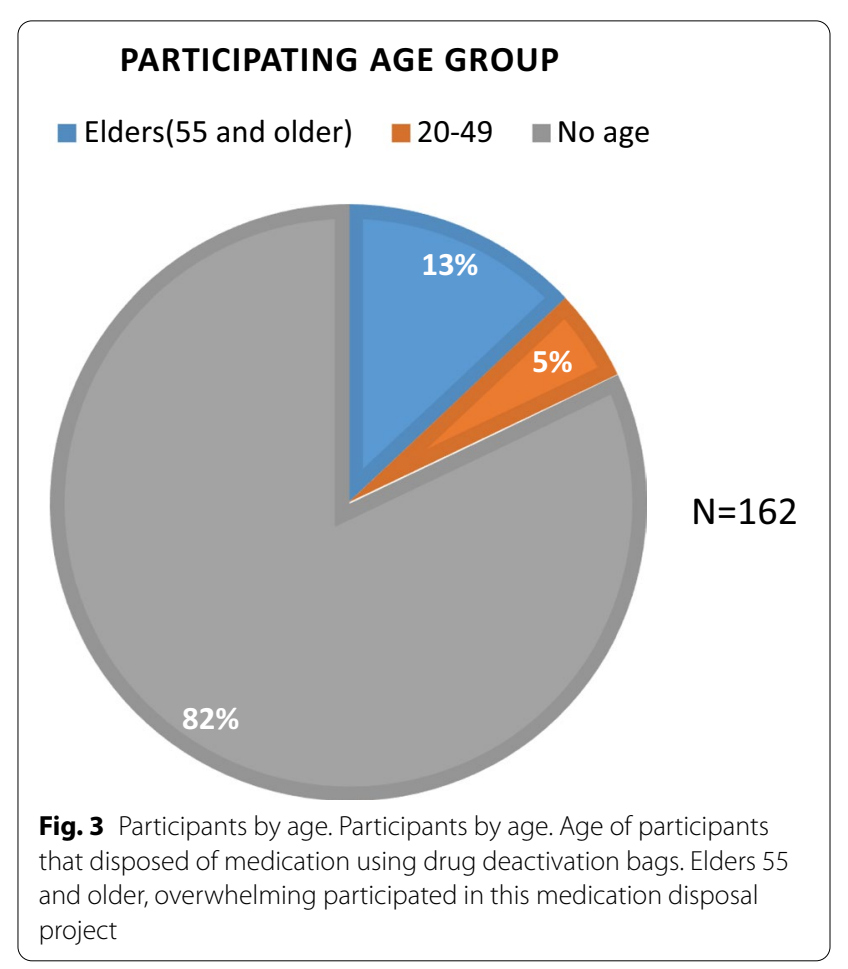

Of persons in the participating 162 households, 82\% $(n=133)$ did not self-report their age; $13 \%(n=21)$ identified as elders (i.e., 55 and above age group); and 5\% $(\mathrm{n}=8)$ identified as adults (i.e., 20-49 age group) (Fig. 3). 
Table 2 Forms of medications disposed of using the deactivation

\begin{tabular}{lllccc}
\hline Setting & $\begin{array}{l}\text { Number } \\
\text { of sites }\end{array}$ & $\begin{array}{l}\text { Number } \\
\text { of drop } \\
\text { boxes }\end{array}$ & $\begin{array}{l}\text { Pounds } \\
\text { disposed } \\
\text { FY19 }\end{array}$ & $\begin{array}{l}\text { Pounds } \\
\text { disposed } \\
\text { FY20 }\end{array}$ & $\begin{array}{l}\text { Total } \\
\text { pounds } \\
\text { disposed }\end{array}$ \\
\hline Rural & 26 & 31 & 1506.73 & 2506.05 & 4012.78 \\
Urban & 3 & 3 & 297.8 & 373.45 & 671.25 \\
Grand total & 29 & 34 & 1804.53 & 2879.5 & 4684.03 \\
\hline
\end{tabular}

Table 3 Forms of medications disposed of using the deactivation

\begin{tabular}{llllll}
\hline Site & $\begin{array}{l}\text { Number } \\
\text { of sites }\end{array}$ & $\begin{array}{l}\text { Number } \\
\text { of drop } \\
\text { box }\end{array}$ & $\begin{array}{l}\text { Pounds } \\
\text { disposed } \\
\text { FY19 }\end{array}$ & $\begin{array}{l}\text { Pounds } \\
\text { disposed } \\
\text { FY20 }\end{array}$ & $\begin{array}{l}\text { Total } \\
\text { pounds } \\
\text { disposed }\end{array}$ \\
\hline Federal & 17 & 20 & 1636.43 & 1730.1 & 3366.53 \\
Tribal & 12 & 14 & 168.1 & 1149.4 & 1317.5 \\
Grand total & 29 & 34 & 1804.53 & 2879.5 & 4684.03 \\
\hline
\end{tabular}

\section{Medication drop boxes}

From October 1, 2018, to September 30, 2020 data were collected from 34 drop boxes located in 29 healthcare facilities. A total of 4684 pounds of medication were collected and disposed of through healthcare facility drop boxes. Ninety percent of the boxes were located in rural settings (Table 2).

Federal healthcare facilities disposed of 3367 pounds of medication during the project period (Table 3 ).

\section{Discussion}

Based on findings from this project, limited knowledge and access to medication disposal can lead to stockpiling medications in the home, potentially leading to increased diversion, theft or abuse. Many communities in rural settings do not have the same access as those in urban settings. Collection differences in rural communities may also be attributed to lack of access to retail pharmacies or other limitations such as lack of available resources for medication disposal.

The two interventions selected for this project were a result of the community engagement process. This led to increased acceptance and participation. Prior to the project, medication disposal options were limited. At completion of the project, multiple options were available and used. Partnering with healthcare facilities contributed to the success of this project due to their convenience and visitation frequency by the community members. Access to disposal in the home environment was also a convenient alternative for rural tribal communities. This project benefited from the multiple partnerships that exist in tribal communities. The $\mathrm{CHR}$ and $\mathrm{PHN}$ programs that conduct home health services were critical to the implementation of the drug deactivation bags in the home environment. These programs were instrumental in providing education for proper medication disposal in addition to identifying recipients of resources made available by state partners.

\section{Limitation}

Data collection instruments designed for this project were not end user friendly. As a result, there were gaps in collecting information regarding types of medications disposed. Moreover, to further validate the findings of this project, additional marketing would be needed to expand the number of participants. Lastly, materials used for this project required external funding and future projects would have to identify funding sources. An additional barrier to replicate this project on a larger scale is to identify community partners to distribute and market project interventions.

\section{Conclusion}

The project assessed distribution of drug deactivation bags and medication drop boxes, which proved to be acceptable options for medication disposal in tribal communities. Community education coupled with access to effective disposal options in the home environment and local healthcare facilities resulted in collection of unused/expired medications and reduced diversion risk. The strategies identified in this project are scalable and easy to replicate to address safe medication disposal.

\section{Abbreviations}

Al/AN: American Indians/Alaska Natives; CHR: Community Health Representatives; DEA: Drug Enforcement Administration; FDA: Food and Drug Administration; FY: Fiscal Year; IHS: Indian Health Services; IPP: Injury Prevention Program; PHN: Public Health Nurses; POC: Point of Contact.

\section{Acknowledgements}

The authors of this article would like to acknowledge the following programs for their assistance during the formative phase of this project: IHS Pharmacy, Tribal Community Health Representatives, Public Health Nursing, Tribal leadership, IHS Injury Prevention Program and Phoenix Area Division of Environmental Health Services.

\section{About this supplement}

This article has been published as part of Injury Epidemiology Volume 8 Supplement 2 2021: Highlights from the Inaugural National Conference on American Indian and Alaska Native IVP: Bridging Science, Practice, and Culture. The full contents of the supplement are available at https://injepijournal. biomedcentral.com/articles/supplements/volume-8-supplement-2.

\section{Authors' contributions}

IA, AT, RM, LA and MS provided substantial contributions to the conception and design of the work; or the acquisition, analysis or interpretation of data for the work; and drafting the work or revising it critically for important intellectual content. DB and WC provided contributions by revising the work critically for important intellectual content. All authors provided final approval of the version to be published and are in agreement to be accountable for 
all aspects of the work in ensuring that questions related to the accuracy or integrity of any part of the work are appropriately investigated and resolved. All authors read and approved the final manuscript.

\section{Funding}

This article submission as part of the Supplement-Proceedings from the 2019 National Conference on Al/AN Violence and Injury Prevention-is funded by the author's Agency, the Indian Health Service, part of the U.S. Department of Health \& Human Services.

\section{Availability of data and materials}

The datasets used and/or analyzed during the current study are available from the corresponding author on reasonable request.

\section{Declarations}

\section{Ethics approval and consent to participate}

Although no personal identifying information was collected during this project, the authors voluntarily elected to obtain written permission from each tribal government to implement, evaluate, and publish the findings of the project. The Phoenix Area Indian Health Service IRB determined no review was necessary and provided a written waiver.

\section{Consent for publication}

Not applicable.

\section{Competing interests}

The authors declare that they have no competing interests.

\section{Author details}

${ }^{1}$ IHS - Western Arizona District Office, 1553 West Todd Drive, Suite 104, Tempe, AZ 85283, USA. ${ }^{2}$ IHS - Phoenix Area Office, 40 North Central Avenue, Suite 600, Phoenix, AZ 85004, USA. ${ }^{3}$ HHS - Eastern Arizona District Office, 5448 S. White Mountain Blvd., Suite 220, Lakeside, AZ 85929, USA. ${ }^{4} 3685$ E. Perkinsville Street, Gilbert, AZ 85295, USA. ${ }^{5}$ HHS - Reno District Office, 1150 Financial Blvd. Suite 500, Reno, NV 89502, USA. ${ }^{6}$ IHS - Rhinelander District Office, 9a South Brown St, Rhinelander, WI 54501, USA. ${ }^{7}$ IHS - Oklahoma Area Office, 701 Market Dr, Oklahoma City, OK 73114, USA.

Accepted: 25 November 2021

Published online: 08 December 2021

\section{References}

Center for Drug Evaluation and Research. Drug disposal: Fda's FLUSH list for certain medicines. U.S. Food and Drug Administration (FDA). n.d. Retrieved February 18, 2021, from https://www.fda.gov/drugs/dispo sal-unused-medicines-what-you-should-know/drug-disposal-fdas-flushlist-certain-medicines.

Defining rural population. 2021. Retrieved February 19, 2021, from https:// www.hrsa.gov/rural-health/about-us/definition/index.html.

McCance-Katz E. 2018 National Survey on Drug Use and Health: American Indian and Alaska Natives (A.I./ANs). Substance Abuse and Mental Health Services Administration (SAMHSA). 2019. https://www.samhsa.gov/data/ release/2018-national-survey-drug-use-and-health-nsduh-releases.

National Center for Injury Prevention and Control, C. 10 leading causes of death, United States 2018, Am Indian/AK Native, Both Sexes. U.S. Food and Drug Administration (FDA). n.d. Retrieved December 7, 2019, from https://webappa.cdc.gov/cgi-bin/broker.exe.

National Institutes of Health. Overdose death rates. 2020. Retrieved February 18, 2021, from https://www.drugabuse.gov/drug-topics/trends-statistics/ overdose-death-rates.

Service, I. H. U.S. Department of Health and Human Services. 2020. Retrieved from Indian Health Service: https://www.ihs.gov/.

Wilson N, Kariisa M, Seth P, Smith H IV, Davis NL. Drug and opioid-involved overdose deaths_United States, 2017-2018. MMWR Morb Mortal Wkly Rep. 2020;69:290-7. https://doi.org/10.15585/mmwr.mm6911a4externa licon.

\section{Publisher's Note}

Springer Nature remains neutral with regard to jurisdictional claims in published maps and institutional affiliations.
Ready to submit your research? Choose BMC and benefit from:

- fast, convenient online submission

- thorough peer review by experienced researchers in your field

- rapid publication on acceptance

- support for research data, including large and complex data types

- gold Open Access which fosters wider collaboration and increased citations

- maximum visibility for your research: over 100M website views per year

At BMC, research is always in progress.

Learn more biomedcentral.com/submissions 